\title{
Achievements in Astronomical Video Data Collection
}

Paul D. Maley

International Occultation Timing Association, 15807 Brookvilla, Houston, TX 77059, U.S.A.

Observation by amateurs has been traditionally viewed with some suspicion by the professional astronomical community, especially in the case of transient events(1). For example, data from variable star monitoring where information from many observers can be integrated over long intervals, have a higher weight than visual observations of an asteroid occultation. Serious amateurs who choose to contribute to the science face a continuing challenge to maintain the highest standards.

In an effort to upgrade the quality of information transmitted to professional institutions, we have experimented since 1984 with a baseline design of mobile video equipment which can be coupled to objective lenses ranging from $28 \mathrm{~mm}$ to $625 \mathrm{~mm}$ aperture (2).

A composite baseline system consists of the objective which passes light through to the intensifier, which then continues to a relay lens that transfers the image into a television camera. Once in video format the signal is output to a monitor and video cassette recorder. The system we use now consists of a RCA Ultricon camera, Litton M841 Pocketscope image intensifier, a relay lens, and celestron 8 telescope. The objective has been interchanged with $100 \mathrm{~mm}$ length lenses depending on the object under analysis.

We find that video achieves a level of accuracy better than the human eye but somewhat less than photometry. Time resolution is .03 seconds, consistent with a 30 frame/second recording rate, while a character generating 
accessory can display the time to either .1 or .01 seconds. Video offers distinct advantages of reusable reconding material, playback and reviewing, slow motion or rapid frame advancement. These are characteristics which cannot be provided by photometric methods. Seeing effects may be isolated by comparison with pixel elements in other parts of the field not under study, such as the moon's surface in the case of lunar occultations and other stars, as with minor planet occultations.

Power can be supplied from the mains (110V AC, 60cps) or from $12 \mathrm{~V}$ DC creating the ability to transport the hardware for field useage. However in mid 1987 portable video cassette recorders are rapidly disappearing from the market place and are being replaced by the camcorder models which at present are not adaptive to low light astronomical use. The celestron 8 system permits stellar images to magnitude 10.5 to be recorded under the best sky conditions using an $18 \mathrm{~mm}$ aperture intensifier tube. With a $25 \mathrm{~mm}$ tube, sensing to +11 is possible, though the price may more than double for the increased size of that component. Performance is impacted if the tube is not new, contains blemishes, or is of an earlier generation. A fast "matched" relay lens is essential to maintain the signal level and field symmetry as the image is passed through the optical train(3). Intensification is not required on such targets as solar eclipses and occultations of stars brighter than 5th magnitude.

We have worked on the following aerospace and astronomical applications of vi deo to the science in an effort to demonstrate the ability of amateurs to contribute useful data on a variety of subjects (4). our first use of a intensifier in 1972 accidentally discovered a brief burst of activity from the Giacobinid radiant of meteors below naked eye threshhold (5).

ECLIPSES: high precision detailed imagery of Baily's Beads have been obtained during the annular eclipse of May 30, 1984 (Atlanta, Georgia USA) and from the total eclipse of November 23, 1984 (Kwikila, Papua New Guinea) 
using unintensified video and projection techniques(6). Direct imagery of a one-second total-annular eclipse was achieved in Gabon on March 29, 1987. These data are being employed in the U.S. Naval Observatory solar radius variation study project, in which IOTA is a major participant.

OCCULTATIONS: numerous grazing lunar occultations have been observed since 1984 including those of Alpha Virginis and Eta Tauri in 1987. Clear definition of instantaneous and slow disappearances and reappearances have been obtained thorough evaluation of the exact number of video frames required for each phenomenon. The video records also serve as key anchors in reducing the more numerous visual observations. Duplicity of close components have also been recognized in the case of stars whose separations are 0.1 arcsecond or greater. A video record of the grazing occultation of Alpha Libra from Sudan during the May 4, 1985 total lunar eclipse was completed with a similar system (100mm $\mathrm{f} / 10$ objective). Analysis is underway to determine if this event will produce the most accuracte ground based determination yet of the lunar polar diameter(7). As many as 5 video systems have been simultaneously used on a single graze event.

Because of the rarity of asteroid occultation events, several have been attempted by us in which any clear reduction in magnitude of 0.3 or more would have been detectable, but none have yet been seen. Other IOTA observers have recorded at least 2 minor planet cocultations with a portable system of similar design $(8,8 \mathrm{~A})$.

COMEIS: Stellar appulses to comet $\mathrm{P} / \mathrm{Hall}$ ley have been recorded on video on several cocasions. While no occultation was seen, the lack of an outage allowed an upper limit to be set on the dust density in the vicinity of the nucleus prior to the Halley spacecraft fly-by. The comet was also video taped from the northern Chilean desert in April 1986, with a $12.5 \mathrm{~cm} \mathrm{f} / 2$ objective; and a stellar appulse to Comet GiacobinniZinner has also been recorded. However our optical search for new comets 
conducted between 1975-1987 has been unsuccessful. We have employed low light television only within the last 3 years to assist in acquisition. Although this search has not achieved discovery of a comet, we conclude that image tubes fail to integrate enough light to permit distinguishing diffuse comets from point sources in order for them to serve as comet locators.

AEROSPACE EVENTS: Man-made space targets have proven more frequent and productive in which to apply the baseline system. A recent analysis of the sunlight reflecting behavior of a number of Soviet spacecraft have aided in the proof needed to demonstrate that claims of a new astrophysical discovery (the so-called Aries or Perseus Flasher) are more likely to be glints from artificial earth satellites (9). Light curves have been constructed using video recordings that were instrumental in the determination of the optimum rendezvous attitudes required from which to snare the disabled Westar and Palapa spacecraft in 1984 by a Space Shuttle crew. The reflectivities were obtained under a variety of phase angles.

This video arrangement has been used to study the reflectivity of over 30 geostationary satellites (with a $31 \mathrm{~cm}$ reflector), and to determine details on the stability and on-orbit behavior of Soviet surveillance craft using a $100 \mathrm{~mm}$ objective (10). our two-year analysis of 14-day Soviet reconaissance satellites discovered key visibility seasons from which to monitor separation of the main body into recoverable capsules and earth resources subsatellites (11). Also in 1985, the NOAA 8 spacecraft suffered an internal explosion. The true rotation rate masked within a complex pattern of light changes was clearly found from our video data, and from that an initial velocity imparted by the explosion.

our video was the only amateur astronomical data source contributed to the Max Plank Institute magnetospheric experiment known as AMPIE in 1984. Imagery which has been frame stacked and enhanced by an offline special processor clearly showed a solar wind shock front developing around a 
barium release. Another outstanding application has been the NASA acceptance of our suggestion to perform a controlled waste water dump from the Space Shuttle at a terminator crossing under dark sky conditions. This created a spectacular naked eye artificial comet seen by thousands of ground observers; it unexpectedly proved that contamination from routine expulsion of waste products from manned space vehicles could prove to be detrimental to astronomical experiments conducted in real-time or a number of orbits later. A plume $28 \mathrm{~km}$ long and $1.5 \mathrm{~km}$ wide was shown to exist, which later actually recontacted the Shuttle! our video has been incorporated in an official Detailed Test Objective in which NASA hopes to study future effluence behavior under a variety of attitudes (12) .

ours was the first video taken of a Space Shuttle reentry, and it verified a theoretical chemiluminescent effect, which proved that a blunt nosed body entering the atmosphere causes a residual glow in its wake (13). This is extremely important in understanding the behavior of the upper atmosphere. One unusual phenomenon created by the 1908 Tunguska encounter was the highly brightened night sky which persist for several days following the event. A discovery of primary and secondary fireballs attending the Space Shuttle at high Mach numbers was evidenced on our tape. The most dramatic application came on April 6, 1984 as we successfully monitored the reentry of the Shuttle external fuel tank from the slopes of the Mauna Kea volcano in Hawaii (14). A complete detailed evolution of each major fragment was clearly recorded and its trajectory determined.

The baseline video system can be operated by one person and most components may be hand-carried onto a commerical aircraft. It has been tried and proven under a variety of conditions. While video hardware is not consistent in terms of frequency and signal characteristics, manufacturers do supply VCR, camera and monitor components in SECAM and PAL-B. A video tape recorded on SECAM cannot play on a recorder/monitor which is designed for 
the American NISC, so that the electronics must be considered with this limitation in mind. While tape formats can be interchanged, the reconded signals must be converted between systems; this can be an expensive drawback.

We encourage the proliferation of this type of technology into the hands of the international amateur astronomical community so that they may apply it to the science. The ultimate goals may be educational or research oriented but amateurs will find an increasing respect as their tools lift their status and ultimately their treatment as co-investigators from a heretofore lower case role.

Sources in Europe for image intensifier assemblies and relay lenses are from Litton Precision Productions International, Inc. Inquiries may be made to this company through the following offices:

58 Rue Pottier

F 78150 Le Chesnay FRANCE

Oberfohringer Strasse 8 D-8000 Munich 80

FRG

6 First Avenue Globe Park, Marlowe

Bucks, SL7 IYA

UK

We would like to gratefully acknowledge Litton Electron Devices, Tempe, Arizona USA for the use of a prototype image intensifier for testing purposes, and to Peter Manly for hardware design assistance and review of this paper.

\section{REFERENCES}

1. H. Reitsema, SCIENCE, 1979, Vol. 205, p. 185.

2. P. D. Maley, PULSAR, September-October 1985, No. 656, p.220.

3. P. Manly, "Coupling Intensifiers to Television Sensors" (submitted to the Eighth Annual IAPPP Symposium, February 1987).

4. P. D. Maley, SPACE EDUCATION, Vol. 1, No. 9, May 1985, p.415

5. B. Marsden, IAUC 2452, October 20, 1972.

6. P. D. Maley, L'ASTRONOMIA, September 1986, No. 58, p. 54 .

7. D. Dunham, OCCULTATION NEWSLETIER, Vol. III 1985, p.246.

8. D. Dunham, OCCULTATION NEWSIETTER, Vol. III 1984, p. 182. 
8A. D. Dunham, OCCUITATTION NEWSLETTER, Vol. III 1984, p. 102.

9. P. D. Maley, ASTROPHYSICAL JOURNAL LETTERS, Vol. 317, L39-LA4, 1987.

10. N.L.Johnson, THE SOVIET YEAR IN SPACE 1984, 1985, p.33.

11. P. D. Maley, PROCEEDINGS OF THE 1985 SYMPOSIUM ON SPACE C3, USAF Academy, Colorado Springs, May 12, 1985, p.133.

12. P. D. Maley and L. Leger, NASA FLIGHT TEST AND SUPPLENENTAL OBJECTIVES DOCUMENT, DTO \#0330., 1986.

13. P. D. Maley, ACTA ASIRONAUTICA, Vol. 12, No. 10, 1985, p. 755.

14. P. D. Maley, SPACEFLIGHT, December 1984, Vol. 26, No. 12, p.446. 